\title{
Minireview
}

Experimental
Nephrology

\section{Recent Advances in Animal Models of Diabetic Nephropathy}

\author{
Boris Betz $^{\text {a }}$ Bryan R. Conway ${ }^{b}$ \\ Centres for ${ }^{\mathrm{a}}$ Inflammation Research and ${ }^{\mathrm{b}}$ Cardiovascular Science, University of Edinburgh, Edinburgh, UK
}

\section{Key Words}

Diabetic nephropathy · Background strain · Hypertension ·

Transcriptomic profiling

\begin{abstract}
Diabetic nephropathy (DN) is the single most common cause of end-stage kidney disease. Therefore, it is imperative that novel therapies are developed. Progress has been hindered, however, by the lack of robust animal models. In the current review we describe recent advances in the field, including the impact of background strain, hypertension and transcriptomic profiling. While the $\mathrm{C} 57 \mathrm{BL} / 6 \mathrm{~J}$ strain is relatively resistant to $D N$, the FVB strain appears more susceptible and Ove26 and db/db mice on this background may be useful in modelling types 1 and 2 DN, respectively. Black and tan, brachyury (BTBR) mice deficient for the leptin receptor (ob/ ob) develop many of the pathological features of human DN and, remarkably, treatment with exogenous leptin ameliorates hyperglycaemia, albuminuria and glomerulosclerosis. Hypertension plays a key role in the progression of human DN and exacerbates nephropathy in diabetic rodents. Endothelial nitric oxide synthase deficiency $\left(\mathrm{eNOS}^{-/}\right)$results in moderate hypertension and the development of nodular glomerulosclerosis and hyaline arteriosclerosis in streptozotocin-induced diabetic C57BL/6J mice. In Cyp1a1mRen2 rats, renin-dependent hypertension synergises with streptozotocin-induced hyperglycaemia to produce a 500 -fold in-
\end{abstract}

crease in albuminuria, glomerulosclerosis and tubulointerstitial fibrosis. Renal transcriptional profiling suggests that many of the gene expression changes observed in human DN are replicated in eNOS ${ }^{-/}$mice and Cyp1a1mRen2 rats. Despite these advances, no model faithfully recapitulates all the features of human DN and further refinements are required. In the interim, it is likely that researchers may use publically available transcriptomic data to select the most appropriate model to study their molecule or pathway of interest.

(c) 2014 S. Karger AG, Basel

Diabetic nephropathy (DN) is one of the most feared complications of diabetes. It remains the single largest cause of end-stage renal disease in the Western world, having a devastating impact on patients with diabetes and placing a major burden on health care resources [1]. Therefore, it is imperative that novel therapies are developed to retard the progression of the disease. Animal models may be useful in dissecting the pathogenesis of the disease and for testing novel therapies. However, their utility in DN research has been constrained by the fact that most models fail to recapitulate important functional and structural features of advanced human disease [2, 3]. This may partly explain why therapies that have shown benefit in animal models have often proven less effective in human DN.

\section{KARGER}

E-Mail karger@karger.com

www.karger.com/nee (c) 2014 S. Karger AG, Basel

$1660-2129 / 14 / 1264-0191 \$ 39.50 / 0$
Dr. Bryan Conway

Room W3.06, Centre for Cardiovascular Science

University of Edinburgh, Queen's Medical Research Institute

47 Little France Crescent, Edinburgh EH16 4TJ, Scotland (UK)

E-Mail bryan.conway@ed.c.uk 


\section{Animal Models Do Not Faithfully Replicate Human DN}

The nephropathy subcommittee of the Animal Models of Diabetic Complications Consortium (AMDCC) has published the following validation criteria for rodent models of DN based on the clinical and pathological features of human DN [4]: (1) $>50 \%$ decrease in renal function, (2) $>10$-fold increase in albuminuria and (3) pathological features including advanced mesangial matrix expansion (+/- nodules), thickening of the glomerular basement membrane, arteriolar hyalinosis and tubulointerstitial fibrosis. An ideal model of DN would display all of these criteria; however, no current model entirely satisfies them [2]. Another important consideration that has been the focus of recent attention is whether the pathophysiological pathways induced in the kidney in animal models reflect that observed in human DN [5]. This is likely to be a key factor in ensuring successful clinical translation of therapies that have proven beneficial in experimental DN.

In attempts to replicate human DN experimentally, models of both types 1 and 2 diabetes have been employed [4]. Type 1 models include induction of hyperglycaemia by the pancreatic toxin streptozotocin or the use of genetic models such as the Akita or OVE26 mice, in which mutations in the insulin and calmodulin genes, respectively, result in toxic accumulation of the defective protein, specifically in $\beta$-cells. Type $2 \mathrm{DN}$ is typically modelled using genetically obese rodents that have leptin deficiency (e.g. ob/ob mice) or inactivating mutations in the leptin receptor (e.g. db/db mice, Zucker rats). The degree of renal injury observed in these models depends on the background strain of the animal $[2,4]$. The C57BL/6J mouse strain is relatively resistant to $\mathrm{DN}$, which is inopportune, given that many genetic modifications are performed on this background. Recent advances have focused on accelerating renal injury by the following means: (1) performing targeted knockout of key genes in C57BL/6J mice, (2) identifying alternative strains that are more susceptible to nephropathy or (3) superimposing additional factors that are key to the progression of human disease, such as hypertension.

\section{Endothelial Nitric Oxide Synthase Knockout Mice}

A major advance in the field has been the use of endothelial nitric oxide synthase (eNOS/NOS2) knockout mice. Functional eNOS deficiency is an important pathological feature in human chronic kidney disease [6]. Targeted knockout of the eNOS gene results in accelerated renal injury in $\mathrm{db} / \mathrm{db}$ mice [7] and in streptozotocin-induced diabetes, including in mice on the nephropathy-resistant C57BL/6J background [8]. In both cases the mice exhibit features similar to human DN, including early onset albuminuria, decreased glomerular filtration rate, arteriolar hyalinosis, mesangial expansion, mesangiolysis and nodular glomerulosclerosis. However, only minimal tubulointerstitial fibrosis is observed $[7,8]$, which is of concern given that this is a major prognostic factor in human DN [9].

\section{Susceptibility to DN Is Strain Dependent}

Other recent advances include the identification of strains of mice that are more susceptible to DN. For example, $\mathrm{db} / \mathrm{db}$ mice on the FVB background appear more susceptible to nephropathy than equally obese and hyperglycaemic C57BL/6J mice [10]. In addition, OVE26 mice on the FVB background exhibit nodular glomerulosclerosis and $>10$-fold increase in albuminuria by 6 months of age [11]. Furthermore, as it is for most strains of mice, unilateral nephrectomy in diabetic OVE26 mice accelerates many features of DN, including albuminuria, inflammatory cell infiltration, fibrosis and changes in gene expression [12]. However, the advantages conferred by uninephrectomy must be weighed against the drawback that the resultant abnormal glomerular haemodynamics may not be representative of the pathophysiology of $\mathrm{DN}$, which may reduce the translational potential.

Another interesting but relatively unfamiliar strain is the black and tan, brachyury (BTBR) mouse, which is naturally insulin resistant. While ob/ob mice on the C57BL/6J background are rarely used in modelling $\mathrm{DN}$ as they develop obesity but only mild diabetes, when the ob/ob mutation is placed on the BTBR strain the mice exhibit sustained hyperglycaemia from an early age and develop pathological features of human $\mathrm{DN}$, including arteriolar hyalinosis, mesangial expansion, mesangiolysis, focal nodular glomerulosclerosis and a reduction in podocyte number [13]. However, the mice exhibit only a moderate (approx. 10-fold) increase in albuminuria, mild interstitial fibrosis and no significant change in serum creatinine by 22 weeks. Furthermore, they exhibit markedly increased mortality beyond 24 weeks of age, which limits their use in modelling DN over longer periods. Importantly, as many genetic modifications are performed on the C57BL/6J strain, the benefit of the increased susceptibility to nephropathy exhibited by less commonly used 
strains must be balanced against the cost and delay incurred in replicating the genetic modifications in or backcrossing onto the alternative strain.

\section{Role of Hypertension in Promoting DN in Human and Animal Models}

Most rodent models remain normotensive, which is of concern given that hypertension is almost ubiquitous in advanced human $\mathrm{DN}$ and that blood pressure control is at least as important as glycaemic control in slowing disease progression [14]. Hypertension may not simply be a consequence of $\mathrm{DN}$ but may also be important in initiating the disease. Patients with diabetes who develop nephropathy are more likely to have a parental history of hypertension [15], suggesting that a tendency towards higher blood pressure increases the risk of subsequent nephropathy. This is supported by observational studies, which demonstrate that early loss of nocturnal dipping of blood pressure precedes the onset of albuminuria [16]. However, perhaps the most remarkable evidence for the pre-eminence of hypertension in mediating progressive $\mathrm{DN}$ is derived from an 'experiment of nature. There are two case reports of patients with longstanding diabetes and coexisting unilateral renal artery stenosis who exhibited no evidence of nephropathy in the kidney downstream of the stenosis despite severe nephropathy in the contralateral kidney [17, 18], suggesting that transmission of hypertension to the kidney is a prerequisite for the progression of DN.

Several rodent models have been employed to illustrate the synergy between hyperglycaemia and hypertension in promoting DN. Increased albuminuria, glomerulosclerosis and, importantly, tubulointerstitial fibrosis have been consistently observed with combined diabetes and hypertension compared with diabetes alone [19-22]. Cognizant of the specific beneficial effects of renin-angiotensin system inhibition in treating nephropathy, researchers have induced hyperglycaemia in the mRen 2 rat, in which a copy of murine renin cDNA is integrated into the genome, leading to renin-dependent hypertension [20]. While diabetic mRen 2 rats develop marked albuminuria and nodular glomerulosclerosis, the model is restricted by the onset of malignant-phase hypertension [23]. This problem has been overcome by the use of the Cypla1mRen2 rat in which the mRen2 gene is under the control of the Cyplal promoter, such that the severity of hypertension may be controlled by adjusting the concentration of indole-3-carbinol in the diet [22]. After 28 weeks of streptozotocin-induced diabetes, in the absence of hypertension, there was a 10 -fold rise in albuminuria but minimal evidence of renal scarring, while concurrent induction of hyperglycaemia and renin-dependent hypertension provoked a 500 -fold increase in albuminuria with moderate glomerulosclerosis and tubulointerstitial fibrosis, all features of moderately advanced human DN. However, not all of the classical features of DN were observed, such as arteriolar hyalinosis, and a significant decline in renal function was not apparent. In addition, while it is now possible to perform targeted gene knockout in rats, it is likely to be several years before the largescale availability of gene knockouts or reporters in mice is recapitulated in rats, and in the interim researchers will have to incur the additional cost of generating their own genetically modified rodents.

\section{Use of Transcriptomic Profiling to Compare Pathways Activated in Human and Experimental DN}

While researchers have long focused on replicating the clinical and histological features of human DN, increased attention has been directed towards assessing whether the pathophysiological pathways activated in human disease are recapitulated in animal models. Technological advances such as the ability to microdissect the glomerular and tubulointerstitial compartments by laser capture microscopy, isolate RNA from formalin-fixed paraffin-embedded tissue and systematically assess gene expression by microarray or RNA sequencing have dramatically increased our understanding of the molecular pathways activated in human DN [24]. Furthermore, much of this information has been made freely available to the nephrology community through web-based interfaces such as Nephromine (www. nephromine.org). Researchers are now employing similar techniques to assess whether key pathways activated in human DN are similarly induced in rodent models [3].

Hodgin et al. [5] systematically compared the glomerular transcriptome in three murine models of DN (streptozotocin-induced diabetes on the DBA/2 background, $\mathrm{db} / \mathrm{db}$ mice and $\mathrm{eNOS}^{-/-} \mathrm{db} / \mathrm{db}$ mice) with that from humans with biopsy-proven early type $2 \mathrm{DN}$. In general, the transcriptomic changes in the murine models more closely resembled the pattern observed in those with low-grade albuminuria, consistent with the fact that the murine models mimic the early stages of DN. Furthermore, the human DN network was more congruent with the eNOS $^{-/-} \mathrm{db} / \mathrm{db}$ mice transcript network than with the other animal models tested, corroborating the clinical and pathological data which suggest that the $\mathrm{NOS}^{-/-}$mouse 
Fig. 1. Schemata outlining differences between classical models of $\mathrm{DN}$ and more recent advances where background strain or concurrent hypertension confers additional susceptibility to nephropathy. STZ = Streptozotocin; $\mathrm{db} / \mathrm{db}=$ leptin receptor mutation; ob/ob = leptin deficiency; Cypla1mRen $2=$ transgenic rats with the murine renin $\mathrm{CDNA}$ inserted under an inducible promoter; GFR = glomerular filtration rate.

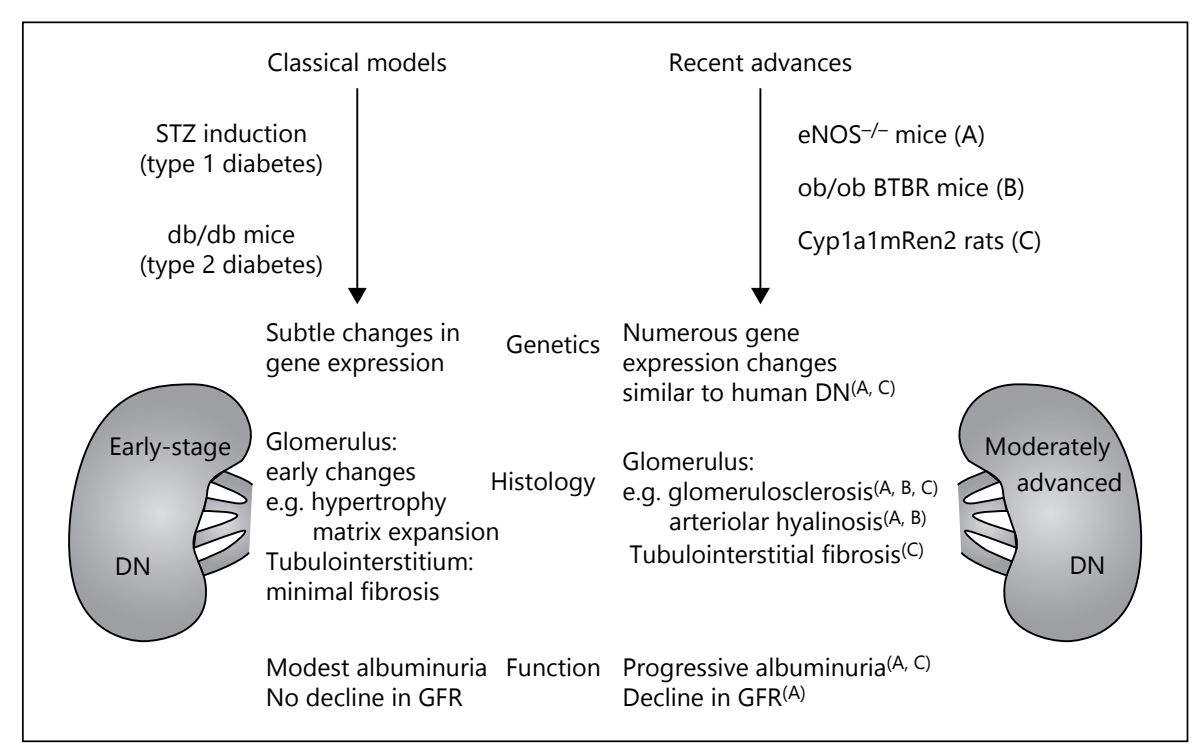

may be a more representative model of human disease [ 7 , 8]. Many pathways that are differentially expressed in the glomerulus in human DN were similarly expressed in just one of the murine models, implying that researchers could use this database to select the most appropriate model for investigating the role of a particular pathway.

Similarly, the pattern of differential gene expression in the renal cortex of hyperglycaemia and hypertensive Cyp1almRen2 rats was compared with that observed in the tubulointerstitium in human DN [22]. Remarkably, 28 weeks of severe hyperglycaemia in isolation resulted in few robust gene expression changes, while the addition of hypertension induced up to $50 \%$ of the gene expression changes observed in human DN. Importantly, the majority of the changes in gene expression were in the same direction in the rats as in humans, in contrast to the discordant pattern that emerged when the transcriptome in murine models was compared with that from patients with more severe proteinuria. The implication of these findings is that researchers must consider which stage of nephropathy they wish to study, with standard murine models reflecting the pathophysiology of early, but not late, DN, while additional relevant injurious stimuli, such as hypertension, may be required to model progressive disease (fig. 1).

\section{Modelling Regression of DN}

While most animal models attempt to mimic progressive $\mathrm{DN}$, it is now recognised that $\mathrm{DN}$ may also regress. Regression of albuminuria, and indeed glomerulosclero- sis and tubulointerstitial fibrosis, has been observed with tight blood pressure and blood glucose control [25]. While this offers hope to those with already moderately advanced disease, the pathways that promote regression remain poorly understood, in part because serial biopsies are rarely performed in patients who are clinically better. Hence, the recent description of regression of albuminuria and glomerular and tubulointerstitial pathology following the administration of leptin in BTBR ob/ob mice may provide the opportunity to dissect the mechanisms of repair in a murine model [26].

\section{Conclusion}

Despite recent advances, no animal model fully meets all of the AMDCC criteria for the ideal model of DN. With increases in our understanding of the molecular pathophysiology of human DN, it is likely that targeting additional genes for knockout, either in isolation or in combination, may refine the animal models, while recognising that complete abrogation of gene expression is rarely observed in human disease. For novel animal models, in addition to a description of existing functional and pathological findings, the transcriptomic changes should be examined for relevance to human disease. Such transcriptomic data should enable researchers to select the most appropriate animal model in which to study their gene/pathway of interest. A more considered approach such as this may increase the likelihood that novel therapies that are efficacious in animal models may translate into the clinic. 


\section{References}

$>1$ System URD: USRDS 2012 Annual Data Report: Atlas of Chronic Kidney Disease and End-Stage Renal Disease in the United States. Bethesda, National Institutes of Health, National Institute of Diabetes and Digestive and Kidney Diseases, 2012.

-2 Brosius FC 3rd, Alpers CE, Bottinger EP, Breyer MD, Coffman TM, Gurley SB, et al: Mouse models of diabetic nephropathy. J Am Soc Nephrol 2009;20:2503-2512.

$>3$ Brosius FC 3rd, Alpers CE: New targets for treatment of diabetic nephropathy: what we have learned from animal models. Curr Opin Nephrol Hypertens 2013;22:17-25.

$\checkmark 4$ Breyer MD, Bottinger E, Brosius FC 3rd, Coffman TM, Harris RC, Heilig CW, et al: Mouse models of diabetic nephropathy. J Am Soc Nephrol 2005;16:27-45.

$>5$ Hodgin JB, Nair V, Zhang H, Randolph A, Harris RC, Nelson RG, et al: Identification of cross-species shared transcriptional networks of diabetic nephropathy in human and mouse glomeruli. Diabetes 2013;62:299-308.

-6 Nakagawa T, Johnson RJ: Endothelial nitric oxide synthase. Contrib Nephrol 2011;170: 93-101.

7 Zhao HJ, Wang S, Cheng H, Zhang MZ, Takahashi T, Fogo AB, et al: Endothelial nitric oxide synthase deficiency produces accelerated nephropathy in diabetic mice. J Am Soc Nephrol 2006;17:2664-2669.

$>8$ Nakagawa T, Sato W, Glushakova O, Heinig M, Clarke T, Campbell-Thompson M, et al: Diabetic endothelial nitric oxide synthase knockout mice develop advanced diabetic nephropathy. J Am Soc Nephrol 2007;18:539550.

$>9$ Gilbert RE, Cooper ME: The tubulointerstitium in progressive diabetic kidney disease: more than an aftermath of glomerular injury? Kidney Int 1999;56:1627-1637.

$>10$ Chua S Jr, Li Y, Liu SM, Liu R, Chan KT, Martino J, et al: A susceptibility gene for kidney disease in an obese mouse model of type II diabetes maps to chromosome 8 . Kidney Int 2010;78:453-462.
11 Yuzawa Y, Niki I, Kosugi T, Maruyama S, Yoshida F, Takeda $\mathrm{M}$, et al: Overexpression of calmodulin in pancreatic beta cells induces diabetic nephropathy. J Am Soc Nephrol 2008;19:1701-1711.

12 Zheng S, Huang Y, Yang L, Chen T, Xu J, Epstein PN: Uninephrectomy of diabetic OVE26 mice greatly accelerates albuminuria, fibrosis, inflammatory cell infiltration and changes in gene expression. Nephron Exp Nephrol 2011; 119:e21-e32.

13 Hudkins KL, Pichaiwong W, Wietecha T, Kowalewska J, Banas MC, Spencer MW, et al: BTBR $\mathrm{Ob} / \mathrm{Ob}$ mutant mice model progressive diabetic nephropathy. J Am Soc Nephrol 2010;21:1533-1542.

14 Mogensen CE: Combined high blood pressure and glucose in type 2 diabetes: double jeopardy. British trial shows clear effects of treatment, especially blood pressure reduction. BMJ 1998;317:693-694.

15 Roglic G, Colhoun HM, Stevens LK, Lemkes $\mathrm{HH}$, Manes C, Fuller JH: Parental history of hypertension and parental history of diabetes and microvascular complications in insulindependent diabetes mellitus: the EURODIAB IDDM Complications Study. Diabet Med 1998;15:418-426.

16 Lurbe E, Redon J, Kesani A, Pascual JM, Tacons J, Alvarez V, et al: Increase in nocturnal blood pressure and progression to microalbuminuria in type 1 diabetes. N Engl J Med 2002;347:797-805.

17 Berkman J, Rifkin H: Unilateral nodular diabetic glomerulosclerosis (Kimmelstiel-Wilson): report of a case. Metabolism 1973;22: 715-722.
18 Beroniade VC, Lefebvre R, Falardeau P: Unilateral nodular diabetic glomerulosclerosis: recurrence of an experiment of nature. Am J Nephrol 1987;7:55-59.

19 Cooper ME, Allen TJ, Macmillan P, Bach L, Jerums G, Doyle AE: Genetic hypertension accelerates nephropathy in the streptozotocin diabetic rat. Am J Hypertension 1988; 1 : 5-10.

20 Kelly DJ, Wilkinson-Berka JL, Allen TJ, Cooper ME, Skinner SL: A new model of diabetic nephropathy with progressive renal impairment in the transgenic (mRen-2)27 rat (TGR). Kidney Int 1998;54:343-352.

21 Janssen U, Riley SG, Vassiliadou A, Floege J, Phillips AO: Hypertension superimposed on type II diabetes in Goto Kakizaki rats induces progressive nephropathy. Kidney Int 2003;63: 2162-2170.

$>22$ Conway BR, Rennie J, Bailey MA, Dunbar DR, Manning JR, Bellamy CO, et al: Hyperglycemia and renin-dependent hypertension synergize to model diabetic nephropathy. J Am Soc Nephrol 2012;23:405-411.

23 Hartner A, Cordasic N, Klanke B, Wittmann M, Veelken R, Hilgers KF: Renal injury in streptozotocin-diabetic Ren2-transgenic rats is mainly dependent on hypertension, not on diabetes. Am J Physiol Renal Physiol 2007; 292:F820-F827.

24 Lindenmeyer MT, Kretzler M, Boucherot A, Berra S, Yasuda Y, Henger A, et al: Interstitial vascular rarefaction and reduced VEGF-A expression in human diabetic nephropathy. J Am Soc Nephrol 2007;18: 1765-1776.

25 Fioretto P, Steffes MW, Sutherland DE, Goetz FC, Mauer M: Reversal of lesions of diabetic nephropathy after pancreas transplantations. New Engl J Med 1998;339:69-75.

-26 Pichaiwong W, Hudkins KL, Wietecha T, Nguyen TQ, Tachaudomdach C, Li W, et al: Reversibility of structural and functional damage in a model of advanced diabetic nephropathy. J Am Soc Nephrol 2013;24:10881102.
Recent Advances in Animal Models of Diabetic Nephropathy
Nephron Exp Nephrol 2014;126:191-195 DOI: $10.1159 / 000363300$ 